EESTI NSV TEADUSTE AKADEEMIA TOIMETISED. 28. KOIDE KEEMIA. 1979, NR. 2

ИЗВЕСТИЯ АКАДЕМИИ НАУК ЭСТОНСКОЙ ССР. ТОМ 28 ХИМИЯ. 1979, № 2

\title{
ИССЛЕДОВАНИЕ И РАСЧЕТ РАВНОВЕСИЯ ЖИДКОСТЬ-ПАР В СИСТЕМАХ, СОДЕРЖАЩИХ Н-АЛКИНЫ
}

\author{
Представлена О. Эйзеном
}

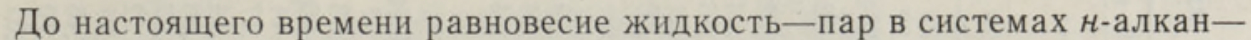
$\boldsymbol{H}$-алкин $\mathrm{C}_{6}$ и $\mathrm{C}_{7}$ изучалось лишь в изотермических условиях [ $\left.{ }^{1}\right]$.

Нами исследовались системы н-октан-октин-1, $H$-нонан-нонин-3 и нонен-3-транс-нонин-3. Основная цель работы заключалась в проверке возможности расчета полных данных равновесия жидкость-пар по концентрационной зависимости температур кипения $t=f(x)$ при постоянном давлении (заданные давления: 760 , 600,400 и 200 мм рт. ст.). Опыты проводились в полумикроэбуллиометре, конструкция которого описана в $\left[{ }^{2}\right]$. Полученные результаты представлены в табл. 1.

Состав пара $y_{i}$ рассчитан путем интегрирования уравнения ДюгемаМаргулеса методом Рунге-Кутта (для $P=$ const):

$$
d y_{1}=2,303 \frac{y_{1}\left(1-y_{1}\right)\left[\frac{B_{1} x_{1}}{\left(t+c_{1}\right)^{2}}+\frac{B_{2} x_{2}}{\left(t+c_{2}\right)^{2}}-\frac{\Delta H_{\mathrm{cM}}}{R T^{2}}\right]}{\left(x_{1}-y_{1}\right)} d T .
$$

Константы уравнения Антуана, использованные для расчетов, определены на основе наших данных по $P-t$ (табл. 1).

На примере системы $\boldsymbol{H}$-октан-октин-1 при нормальном давлении мы попытались выяснить влияние на результаты расчета состава паровой фазы: 1) члена уравнения (1), содержащего величину теплоты смешения $\Delta H_{\mathrm{cм}}$, и 2) поправки на неидеальность паровой фазы.

Теплоты смешения системы $н$-октан-октин- 1 при 25,35 и $45{ }^{\circ} \mathrm{C}$ определены в дифференциальном автоматическом калориметре ДАК 1-1 и скоррелированы при помощи уравнения Редлиха-Кистера:

$$
\frac{\Delta H_{\mathrm{cm}}}{x_{1} x_{2}}=H_{1}+H_{2}\left(x_{1}-x_{2}\right)+H_{3}\left(x_{1}-x_{2}\right)^{2} \text {. }
$$

Константы уравнения (2) даны в табл. 2. Относительное расхождение экспериментальных и расчетных данных не превышает $4 \%$.

Расчет состава пара системы $\boldsymbol{H}$-октан-октин-1 проводился с учетом

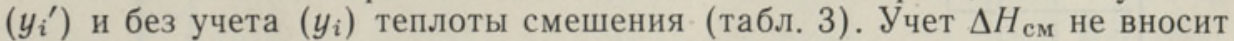
существенных изменений в результаты расчета состава пара. К аналогичным выводам пришли авторы $\left[{ }^{3}\right]$. Поэтому для остальных систем состав паровой фазы был рассчитан по приближенному уравнению (1) без члена, содержащего $\Delta H_{\mathrm{cm}}$. 


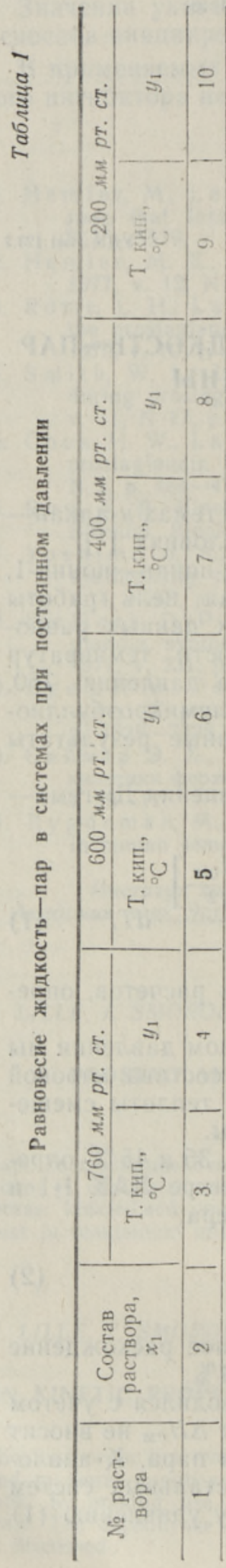

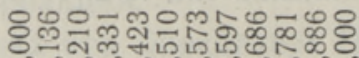 0000000000}

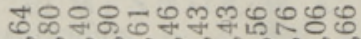
ए๘ఐ

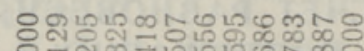 응} ज़

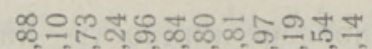

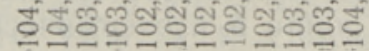
๓ీ

$\infty$

范 $\overrightarrow{\mathrm{T}}$

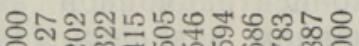
$00000000000=$ 11 u

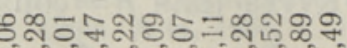

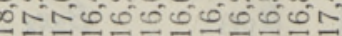

只

เ్ల $\overline{0}$

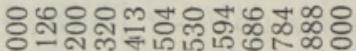 $00^{\circ} 0^{\circ} 0^{\circ} 0^{\circ} 0^{\circ} 0^{\circ} 0^{\circ}=$}

ஸे

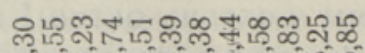

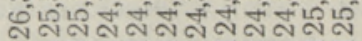
II $\bar{\nabla}$

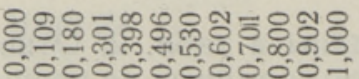

-

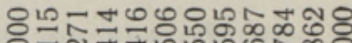 $0.000000000-$}

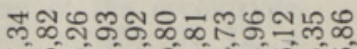

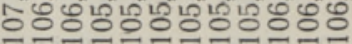

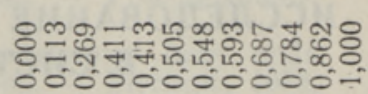

$\underline{0}$

ชㅇำำㅇำำำ

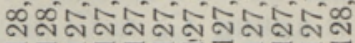
II ตี

ฟิ 至 iI

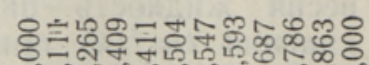
$00^{\circ} 00^{\circ} 0^{\circ} 00^{\circ} 000-$ 푼

离 $\overrightarrow{\mathrm{N}}$ 뜬 ల్ กู

ลำลำั

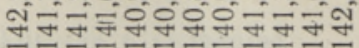
ज

8

용

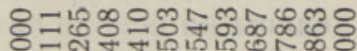
$0.000000000=$ क्

लำ

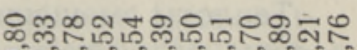
คำ

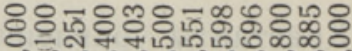
$00000000000=$

-Nm+น 


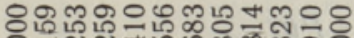

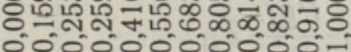

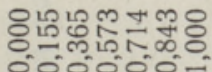

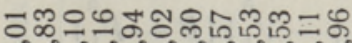

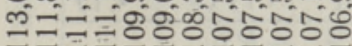

రำษ ल=ํํํํํำ

i⿱宀㠯

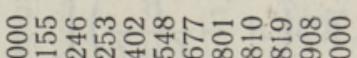

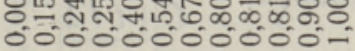
॥ ง

* 응ํํํํํำ융 $000000-$ ง

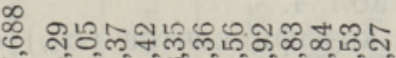

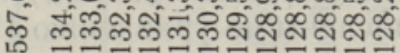
官 II ผั

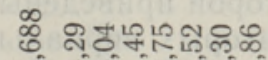

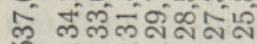

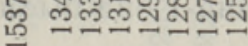
II ๓ี

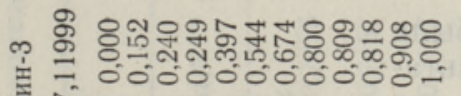

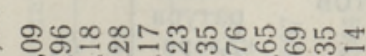

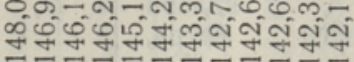
II ज

\%

令

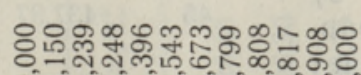
$0000000000-$ क्ष

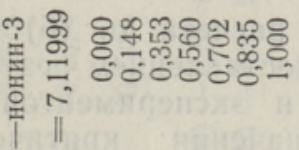

xั

(1) 욘ำ $=8$

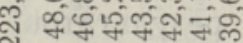
ज

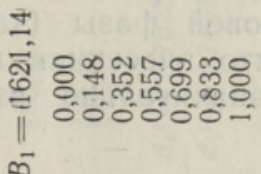

ڤ

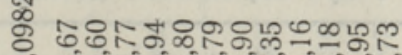

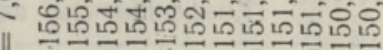
$\bar{\tau}$

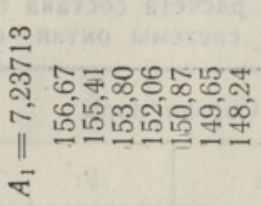

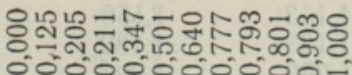


Поправка на неидеальность паровой фазы вычислена по уравнению:

$$
\gamma_{i}=\frac{P y_{i}}{P_{i}^{0} x_{i}} Z_{i}
$$

где

$$
Z_{i}=\exp \frac{\left(B_{i i}-V_{i}^{0}\right)\left(P-P_{i}^{0}\right)}{R T}
$$

и

$$
P_{i}^{0}=\exp \left[2,303\left(A-\frac{B}{C+t}\right)\right]
$$

Значения молярных объемов компонентов $\left(V_{i}{ }^{0}\right)$ были рассчитаны на основе данных о температурной зависимости плотности чистых веществ, уравнения которой приведены в табл. 4.

Значения вторых вириальных коэффициентов $\left(B_{i i}\right)$-октана даны в $\left[{ }^{4,5}\right]$. Для октина-1 они были вычислены по уравнению [ $\left.{ }^{6}\right]$ :

$$
B_{i i}=\frac{R T_{c}}{P_{c}}\left(0,197-0,013 T_{r}-\frac{0,4}{T_{r}}-\frac{0,146}{T_{r}^{3,27}}\right) .
$$

Возможность расчета по (6) была проверена сопоставлением результатов расчета и экспериментов для $\boldsymbol{H}$-октана. Значения критических температуры и давления октина-1 определены методом инкрементов $\left[{ }^{5}\right]$.

Сравнение результатов расчета коэффициентов активности системы $\mu$-октан-октин-1 с учетом $\left(\gamma_{i}{ }^{\prime}\right)$ и без учета $\left(\gamma_{i}\right)$ поправки на неидеальность паровой фазы (табл. 3) показывает, что внесенная поправка лишь незначительно изменяет

\begin{tabular}{|c|c|c|c|}
\hline $\begin{array}{c}\text { Темпе- } \\
\text { ратура } \\
\text { калори- } \\
\text { метра, } \\
{ }^{\circ} \mathrm{C}\end{array}$ & $H_{1}$ & $H_{2}$ & $H_{3}$ \\
\hline $\begin{array}{l}25 \\
35 \\
45\end{array}$ & $\begin{array}{l}489,87 \\
470,48 \\
437,97\end{array}$ & $\begin{array}{c}26,202 \\
6,0894 \\
41,883\end{array}$ & $\begin{array}{r}-33,064 \\
-63,140 \\
30,963\end{array}$ \\
\hline
\end{tabular}

Константы уравнения РедлихаКистера, кал/моль

Таблица 3

Результаты расчета состава пара и қоэффициентов активности компонентов системы октан-октин-1 при нормальном давлении

\begin{tabular}{r|c|c|c|c|c|c}
\hline \multirow{2}{*}{$\begin{array}{c}\text { No } \\
\text { раст- } \\
\text { вора }\end{array}$} & \multicolumn{2}{|c|}{ Состав пара } & \multicolumn{4}{c}{ Коэффициенты активности } \\
\cline { 2 - 6 } & $y_{1}$ & $y_{1}^{\prime}$ & $\gamma_{1}$ & $\gamma_{1}^{\prime}$ & $\gamma_{2}$ & $\gamma_{2}^{\prime}$ \\
\hline & & & & & & \\
1 & 0,0000 & 0,0000 & 1,188 & 1,186 & 1,000 & 1,000 \\
2 & 0,1258 & 0,1257 & 1,153 & 1,152 & 1,003 & 1,002 \\
3 & 0,2002 & 0,2001 & 1,133 & 1,132 & 1,006 & 1,004 \\
4 & 0,3197 & 0,3195 & 1,099 & 1,097 & 1,016 & 1,013 \\
5 & 0,4132 & 0,4130 & 1,073 & 1,070 & 1,029 & 1,026 \\
6 & 0,5035 & 0,5035 & 1,052 & 1,050 & 1,044 & 1,043 \\
7 & 0,5300 & 0,5300 & 1,041 & 1,040 & 1,054 & 1,052 \\
8 & 0,5936 & 0,5937 & 1,030 & 1,028 & 1,072 & 1,069 \\
9 & 0,6861 & 0,6863 & 1,016 & 1,014 & 1,099 & 1,096 \\
10 & 0,7836 & 0,7837 & 1,007 & 1,006 & 1,128 & 1,125 \\
11 & 0,8877 & 0,8877 & 1,003 & 1,002 & 1,157 & 1,156 \\
12 & 1,0000 & 1,0000 & 1,000 & 1,000 & 1,210 & 1,208
\end{tabular}




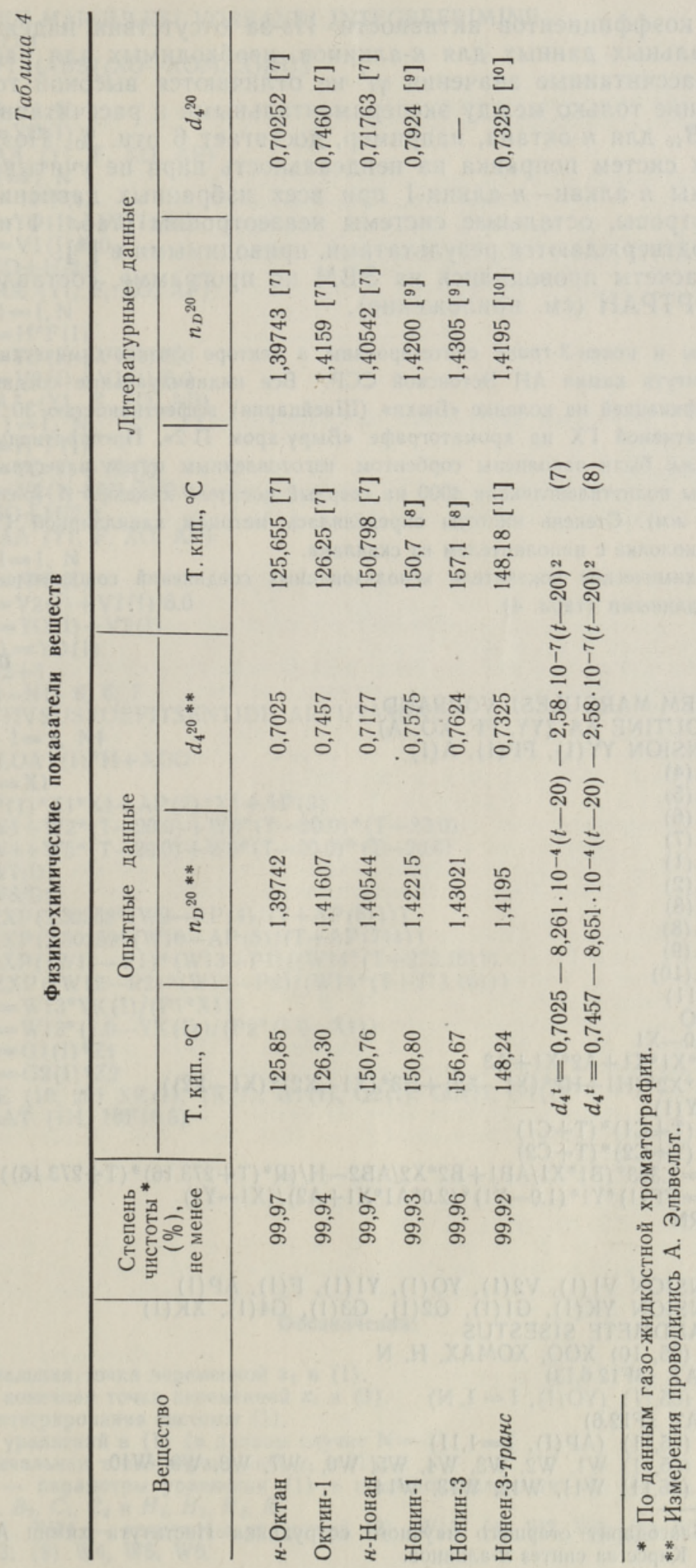


значение коэффициентов активности. Из-за отсутствия надежных экспериментальных данных для $\boldsymbol{H}$-алкинов, необходимых для расчета поправки, рассчитанные значения $\gamma_{i}^{\prime}$ не отличаются высокой точностью. Расхождение только между экспериментальными и рассчитанными значениями $B_{i i}$ для остальных систем поправка на неидеальность пара не учитывалась.

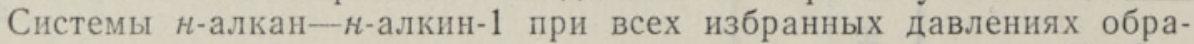
зуют азеотропы, остальные системы неазеотропны (табл. 1 и 3 ). Эти данные подтверждаются результатами, приводимыми в [ $\left.{ }^{1}\right]$.

Bсе расчеты проводились на ЭВМ по программе, составленной на языке ФОРТРАН (см. приложение).

н-Алкины и нонен-3-транс синтезированы в секторе физико-химических исследований Института химии АН Эстонской ССР.* Все индивидуальные соединения очищены ректификацией на колонке «Бюхи» (Швейцария) эффективностью 30 ТТ и методом препаративной ГХ на хроматографе «Выру-хром П-2». Препаративные колонки $6 м \times 14$ мя были заполнены сорбентом, изготовленным путем нанесения $15 \%$-ной жидкой фазы полиэтиленгликоля 4000 на твердый носитель хезасорб $A$ (размер частиц $0,250-0,360 \mathrm{M}$ ). Степень чистоты определялась методом капиллярной ГХ (прибор Хром-4) на колонке с наполнителем из сквалана.

Физико-химические показатели нспользованных соединений сопоставлены с литературными данными (табл. 4).

C 1-DUHEM-MARGULESI VORRAND

SUBROUTINE AA (YY, FF, XO, A)

DIMENSION YY(1), FF(1), A(1)

$\mathrm{B} 1=\mathrm{A}(4)$

$\mathrm{B} 2=\mathrm{A}(5)$

$\mathrm{C} 1=\mathrm{A}(6)$

$\mathrm{C} 2=\mathrm{A}(7)$

$\mathrm{A} 1=\mathrm{A}(1)$

$\mathrm{A} 2=\mathrm{A}(2)$

$\mathrm{A} 3=\mathrm{A}(3)$

$\mathrm{H} 1=\mathrm{A}(8)$

$\mathrm{H} 2=\mathrm{A}(9)$

$\mathrm{H} 3=\mathrm{A}(10)$

$\mathrm{R}=\mathrm{A}(11)$

$\mathrm{X} 1=\mathrm{XO}$

$\mathrm{X} 2=1.0-\mathrm{X} 1$

$\mathrm{T}=\mathrm{A} 1 * \mathrm{X} 1 * \mathrm{X} 1+\mathrm{A} 2 * \mathrm{X} 1+\mathrm{A} 3$

$\mathrm{H}=\mathrm{X} 1 * \mathrm{X} 2 *(\mathrm{H} 1+\mathrm{H} 2 *(\mathrm{X} 1-\mathrm{X} 2)+\mathrm{H} 3 *(\mathrm{X} 1-\mathrm{X} 2) *(\mathrm{X} 1-\mathrm{X} 2))$

$\mathrm{Y} 1=\mathrm{YY}(1)$

$\mathrm{AB} 1=(\mathrm{T}+\mathrm{C} 1) *(\mathrm{~T}+\mathrm{C} 1)$

$\mathrm{AB} 2=(\mathrm{T}+\mathrm{C} 2) *(\mathrm{~T}+\mathrm{C} 2)$

$\mathrm{FF}(1)=2.303^{*}\left(\mathrm{~B} 1 * \mathrm{X} 1 / \mathrm{AB} 1+\mathrm{B} 2 * \mathrm{X} 2 / \mathrm{AB} 2-\mathrm{H} /\left(\mathrm{R}^{*}(\mathrm{~T}+273.16)^{*}(\mathrm{~T}+273.16)\right)\right)$

$\mathrm{FF}(1)=\mathrm{FF}(1) * \mathrm{Y} 1 *(1.0-\mathrm{Y} 1) *(2.0 * \mathrm{~A} 1 * \mathrm{X} 1+\mathrm{A} 2) /(\mathrm{X} 1-\mathrm{Y} 1)$

RETURN

END

MAIN

DIMENSION V1 (1), V2(1), YO(1), Y1(1), F(1), AP(1)

DIMENSION YK(1), G1(1), G2(1), G3(1), G4(1), XK(1)

C 2-ALGANDMETE SISESTUS

READ $(05,10)$ XOO, XOMAX, H, N

10 FORMAT (3F12.6,I3)

$\operatorname{READ}(05,11)(\mathrm{YO}(\mathrm{I}), \mathrm{I}=1, \mathrm{~N})$

11 FORMAT (F12.6)

READ (05,11) (AP (I), I= 1,11)

READ (05,11) W1, W2, W3, W4, W5, W6, W7, W8, W9, W10

READ $(05,11)$ W11, W12, W13, W14

* Авторы благодарят старшего научного сотрудника Института химии АН Эстонской ССР Х. Кирсс за синтез $\mu$-алкинов. 


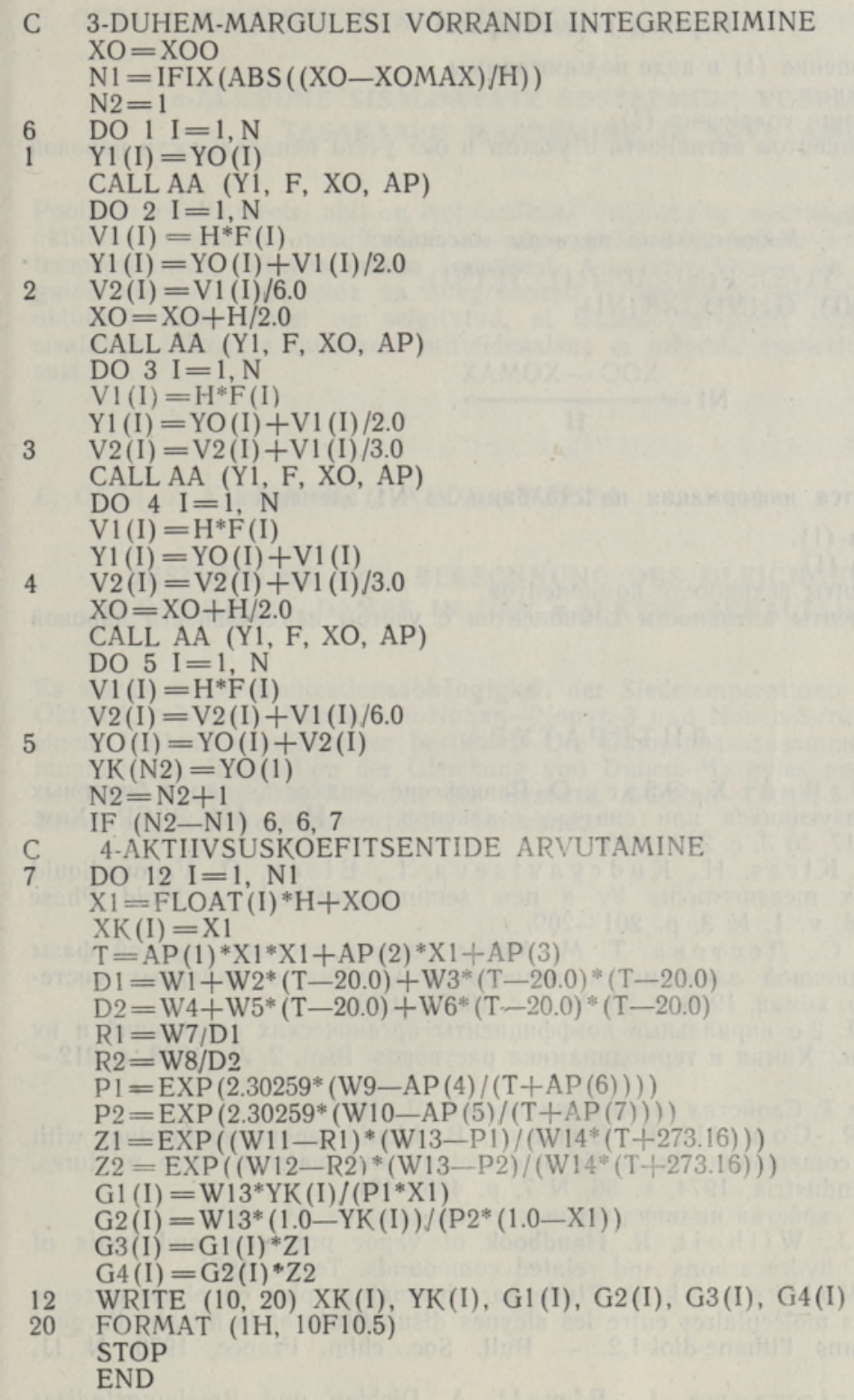

\section{Обозначения:}

ХОО - начальная точка переменной $x_{1}$ в (1).

XOMAX - конечная точка переменной $x_{1}$ в (i1).

$\mathrm{H}$ - шаг интегрирования системы (1).

$\mathrm{N}$ - число уравнений в (1) (в данном случае $\mathrm{N}=1$ ).

YO(11) - начальная точка переменной $y_{1}$ в (1).

$\mathrm{AP}(1-111)$ - параметры уравнения (1) в последовательности: $A_{1}, A_{2}, A_{3}$, константы Антуана $B_{1}, B_{2}, C_{1}, C_{2}$ и $H_{1}, H_{2}, H_{3}, R$.

W1, W2, ..., W14 - константы уравнений (3) W13; (4) W7, W8; (5) W9, W10; (7) W1, W2, W3; (8) W4, W5, W6. 


\section{Перевод комментариев:}

1. Интегрируемое уравнение (1) в виде подпрограммы.

2. Ввод начальных данных.

3. Начало интегрирования уравнения (1).

4. Вычисление коэффициентов активности с учетом и без учета неидеальности паровой фазы.

Минимальные размеры массивов:

$\mathrm{V} 1(\mathrm{~N}), \mathrm{V} 2(\mathrm{~N}), \mathrm{YO}(\mathrm{N}), \mathrm{Y} 1(\mathrm{~N}), \mathrm{F}(\mathrm{N}), \mathrm{AP}(11), \mathrm{YK}(\mathrm{N} 1)$,

G1 (N1), G2(N1), G3(N1), G4(N1), XK(N1), где

$$
\mathrm{N} 1=\frac{\mathrm{XOO}-\mathrm{XOMAX}}{\mathrm{H}}
$$

\section{Выводится информация по столбцам из N1 элементов:}

ХK - переменные $x_{1}$ в (1).

YK - переменные $y_{1}$ в (1).

G1 и G2 - коэффициенты активности компонентов.

G3 и G4 - коэффициенты активности компонентов с учетом неидеальности паровой фазы.

\section{Л И ТЕ Р А Т У Р А}

1. Куд р я це в а Л., В и й т Х., Э й зе н О. Равновесие жидкость-пар в бинарных системах, образующихся при синтезе $\alpha$-алкенов. - Изв. АН ЭССР. Хим. Геол., 1968, т. 17, № 3, с. $242-250$.

2. Mihkelson, V., Kirss, H., Kudryavtseva, L., Eisen, O. Vapour-liquid equilibrium $\mathrm{T}-\mathrm{x}$ measurements by a new semimicromethod. - Fluid Phase Equilibria, 1978, v. 1, N 3, p. 201-209.

3. Буд андев а Л. С., Л естев а Т. М. Метод расчета состава паровой фазы по концентрационной зависимости температур кипения в бинарных системах. - Ж. физ. химии, 1976 , т. 50, № 7, с. $1687-1690$.

4. М аркузин Н. П. 2-е вириальные коэффициенты органических соединений и их смесей. - В кн.: Химия и термодинамика растворов. Вып. 2. Л., 1968, с. 212 238.

5. Рид Р., Ш е р в ул Т. Свойства газов и жидкостей. Л., 1971.

6. Fra ncesconi, R., Comelli, F., C a n e pa, B. Liquid-vapour equilibrium with association of components the acetic acid-acetone and water-pyridine mixtures. - Chimica e industria, 1974 , v. 56, N 7, p. 485-491.

7. Физико-химические свойства индивидуальных углеводородов. М., 1960.

8. Z wolinski, B. J.. Wilhoit, R. Handbook of vapor pressures and heats of vaporization of hydrocarbons and related compounds. Texas, 1971.

9. Queig nec, R., Wojtkowiak, B. Etude par chromatographie en phase gazeuse des associations moléculaires entre les alcynes disubstitués et le nitrate d'argent en solution dans l'ithane-diol-1,2. - Bull. Soc. chim. France, 1970, N 11, p. $3829-3833$.

10. Eisen, O., Kudrjawzewa, L., Elwelt, A. Dichten und Brechungsindices der stellungs- und konfigurationsisomeren n-Alkene. - Z. phys. Chem. (Neue Folge), 1973, Bd. 86, S. 33-42.

11. Michkelson. W. J., Elwelt, A. A., Kudrjawzewa, L. S., Eisen, O. G. Die Druckabhängigkeit der Siedetemperaturen der stellungs- und konfigurationsisomeren $n$-Octene, $n$-Nonene und $n$-Decene. - Monatsh. Chem., 1974, Bd. 105, S $1379-1386$.

Институт химии

Академии наук Эстонской ССР
Поступила в редакцию 10/III 1978 


\section{n-ALKUUNE SISALDAVATE SUSTEEMIDE VEDELIKU-AURU TASAKAALU MÄARAMINE JA ARVUTAMINE}

Poolmikroebulliomeetri abil on isobaarilistes tingimustes määratud süsteemide n-oktaanoktüün-1, n-nonaan-nonüün-1, n-nonaan-nonüün-3 ja noneen-3-trans-nonüün-3 keemistemperatuuride sõltuvus lahuse koostisest. Aurufaasi koostis on arvutatud Duhem-Margulesi võrrandi abil, mida on integreeritud Runge-Kutta meetodil. Süsteemi n-oktaanoktüün-1 näite alusel on selgitatud, et Duhem-Margulesi võrrandi segunemissoojust sisaldava liikme ja aurufaasi mitteideaalsus ei mōjusta oluliselt arvutustulemuste täpsust.

\section{E. OTSA, I. KIRJANEN, L. KUDRJAWZEWA}

\section{BESTIMMUNG UND BERECHNUNG DES GLEICHGEWICHTES VON FLUSSIGKEIT-DAMPF IN DEN $n$-ALKYNE BEHALTENDEN SYSTEMEN}

Es wurde die Konzentrationsabhängigkeit der Siedetemperaturen der Systeme n-OktanOktyn-1, $n$-Nonan-Nonyn-1, n-Nonan-Nonyn-3 und Nonen-3-trans-Nonyn-3 isobar mit einem Halbmikroebulliometer bestimmt. Die Dampfphasezusammensetzung wurde durch nummerische Integration der Gleichung von Duhem-Margules nach Runge-Kutta berechnet. Die Aktivitätskoeffizienten des Systems n-Oktan-Oktyn-1 wurden mit und ohne Benutzung der Regalgaskorrektur berechnet. 